\title{
Airborne gravimetry: An investigation of filtering
}

\author{
Vicki A. Childers*, Robin E. Bell ${ }^{\ddagger}$, and John M. Brozena**
}

\begin{abstract}
Low-pass filtering in airborne gravimetry data processing plays a fundamental role in determining the spectral content and amplitude of the free-air anomaly. Traditional filters used in airborne gravimetry, the $6 \times 20$-s resistor-capacitor (RC) filter and the 300-s Gaussian filter, heavily attenuate the waveband of the gravity signal. As we strive to reduce the overall error budget to the sub-mGal level, an important step is to evaluate the choice and design of the low-pass filter employed in airborne gravimetry to optimize gravity anomaly recovery and noise attenuation. This study evaluates low-pass filtering options and presents a survey-specific frequency domain filter that employs the fast Fourier transform (FFT) for airborne gravity data.

This study recommends a new approach to low-pass filtering airborne data. For a given survey, the filter is
\end{abstract}

designed to maximize the target gravity signal based upon survey parameters and the character of measurement noise. This survey-specific low-pass filter approach is applied to two aerogravimetry surveys: one conducted in West Antarctica and the other in the eastern Pacific off the California coast. A reflight comparison with the West Antarctic survey shows that anomaly amplitudes are increased while slightly improving the rms fit between the reflown survey lines when an appropriately designed FFT filter is employed instead of the traditionally used filters. A comparison of the East Pacific survey with high-resolution shipboard gravity data indicates anomaly amplitude improvements of up to $20 \mathrm{mGal}$ and a $49 \%$ improvement of the rms fit from $3.99 \mathrm{mGal}$ to $2.04 \mathrm{mGal}$ with the appropriately designed FFT filter. These results demonstrate that substantial improvement in anomaly amplitude and wavelength can be attained by tailoring the filter to the survey.

\section{INTRODUCTION}

Unlike all other gravity measurement methods, airborne gravimetry has the potential to recover an accurate gravity field at any place on Earth. In the past 15 years, advancements in precise aircraft positioning with Global Positioning System (GPS) carrier phase data have extended the use of the airborne measurement technique to terrestrial as well as over-water surveys. To date, large regions of the planet remain unmapped because of the limitations of land and marine surveys. With long range aircraft, nearly all the Earth is accessible to airborne surveying.

Improvements in hardware, software, and survey methodology continue to lower the overall error budget for airborne gravity. Although there is no universally agreed-upon method for evaluating data accuracy and anomaly resolution is largely a function of aircraft speed, reported rms error and resolution provide an indication of the technique's accuracy. Recent airborne gravimetric surveys have recovered an rms error of 4-5 mGal at wavelengths of $15-20 \mathrm{~km}$ for the high-speed P-3 Orion aircraft (Brozena et al., 1992) for the high-altitude Greenland survey and 1.8-2.0 mGal for the lower altitude (500-600 m) Arctic Ocean surveys at wavelengths of 8-10 km (Childers et al,, 1997). For surveys conducted aboard the smaller de Havilland Twin Otter aircraft, rms errors of $2.7 \mathrm{mGal}$ are reported at wavelengths of 5-10 km (Brozena, 1994), $2.0 \mathrm{mGal}$ at $4-7-\mathrm{km}$ wavelengths (Olesen and Forsberg, 1997), and $\sim 1 \mathrm{mGal}$ at 3-km wavelengths (Gumert, 1995). Rms agreement of 1.2 and $0.9 \mathrm{mGal}$ at $5-\mathrm{km}$ and $10-\mathrm{km}$ wavelengths, respectively, are reported for data collected from a Cessna 404 (Harrison et al., 1995). Such improvements in measurement accuracy now make a rigorous evaluation of data processing methods appropriate to further reduce the error budget.

In this study, we demonstrate that a carefully designed lowpass filter can have a significant impact on the aerogravimetry

\footnotetext{
Manuscript received by the Editor October 30,1995; revised manuscript received May 26, 1998.

${ }^{*}$ Formerly Department of Earth and Environmental Sciences, Columbia University, Lamont-Doherty Earth Observatory, Palisades, NY 10964; currently Naval Research Laboratory, Code 7420, 4555 Overlook Avenue SW, Washington, DC 20375-5350, E-mail: vicki@qur.nrl.navy.mil. $\Varangle$ Lamont-Doherty Earth Observatory of Columbia University, Route 9W, Palisades, NY 10964-8000. E-mail: robinb@ldeo.columbia.edu. ${ }^{*}$ Naval Research Laboratory, Code 7420, 4555 Overlook Avenue SW, Washington, DC 20375-5350. E-mail: john@qur.nrl.navy.mil. (C) 1999 Society of Exploration Geophysicists. All rights reserved.
} 
data accuracy. The traditional filters used in airborne gravimetry, the $6 \times 20$-s resistor-capacitor (RC) filter and the 300-s Gaussian filter, were inherited from marine gravimetry. These filters heavily attenuate all gravity anomaly frequencies except the zeroth frequency (dc) and significantly attenuate the gravity signal. This study recommends a new approach to low-pass filtering airborne data that better preserves anomaly amplitude and wavelength. The efficacy of this approach is demonstrated with data from two aerogravimetry surveys: a reflight comparison from a survey conducted over the West Antarctic ice sheet, and a comparison of airborne data with marine data off the California coast.

\section{THE AIRBORNE GRAVITY FILTERING PROBLEM}

\section{Signal-to-noise issues in airborne gravimetry}

Isolation of the target gravity signal from the effects of aircraft motion is the primary challenge in airborne gravity. Acceleration of the aircraft along the local vertical, as determined from post-processed GPS carrier phase data, is subtracted from the gravimeter measurement to correct for aircraft vertical motion. The data are then corrected with the Eötvös, free-air, and latitude corrections to yield the free-air gravity anomaly at the aircraft's altitude (Harlan, 1968; e.g., Telford et al., 1990). The free-air anomaly is further corrected for offleveling errors that result when high-amplitude horizontal accelerations from course changes or turbulence drive the gyro-stabilized platform offlevel (Peters and Brozena, 1995).

The nature of the raw gravimeter accelerations and the aircraft vertical accelerations complicate the extraction of the gravity signal from the measurements. Both of these data series can be characterized as broad-band signals with high amplitudes at high frequencies, often ranging as much as $\pm 50000 \mathrm{mGal}$ about the mean for a typical survey line. Spectral analysis confirms that both data types exhibit high power out to the Nyquist frequency (Figure 1a). From a measurement environment with high amplitude noise, the challenge is to extract a gravity signal on the order of tens of milligals.

After correcting for all aircraft-induced accelerations, lowpass filtering is the primary technique for removing residual noise from the free-air anomaly. The success of airborne gravimetry rests upon two fundamental assumptions: first, that the signal dominates the noise in the long wavelengths of the gravity signal waveband; second, that the noise is primarily restricted to shorter wavelengths and can be removed by lowpass filtering. The extreme attenuation required to remove the short wavelength noise can simultaneously significantly attenuate the gravity signal.

\section{Identifying the waveband of the gravity signal}

To design the optimum filter for airborne use, we must determine the gravity signal waveband. Although geologic features span a wide range of wavelengths, large-scale features are often the subject of aerogeophysical studies, and their gravity anomalies occupy the longest wavelengths of the observable waveband. The upper limit of the gravity signal waveband is defined by the shortest wavelength observable from the aircraft platform.

In a noise-free environment, the smallest observable anomaly wavelengths would be determined simply by the aircraft altitude above the anomaly source. As aircraft altitude increases, the amplitude of anomalies falls off as $e^{-2 \pi z / \lambda}$, where $z$ is the distance to the anomaly source and $\lambda$ is the wavelength of the anomaly. In overland flights, $z$ is equivalent to the minimum aircraft elevation above the terrain. Over ice sheets and water, $z$ is the minimum distance between the aircraft and the bedrock. This effect is termed upward continuation (Turcotte and Schubert, 1982), and shorter wavelength anomalies are preferentially attenuated as $z$ increases.

One way to estimate the upper limit of the gravity waveband, or the "anomaly detection threshold," is to determine the wavelength of the smallest point source anomaly observable from the aircraft altitude. We begin by predicting the anomaly over a buried sphere as a simple approximation to shallow geologic structures. The wavelength of this anomaly is a function only of the distance $z_{c}$ from the measurement plane to the center of the sphere (e.g., Telford et al., 1990). The anomaly wavelength, which we will term the "geologic wavelength," is characterized by

$$
\lambda_{g}=1.54 z_{c}
$$
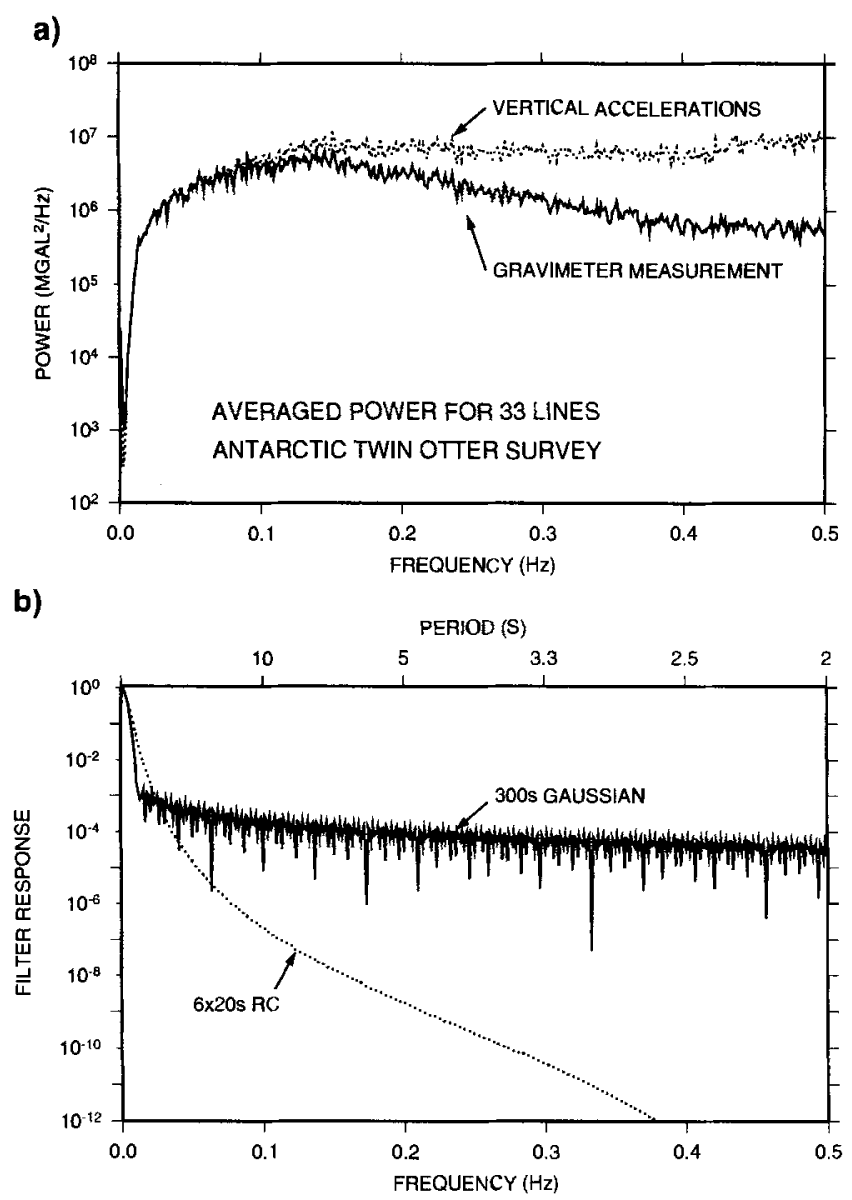

FIG. 1. (a) Power spectra of unfiltered gravimeter measurement and corrected vertical accelerations averaged for 33 survey lines. The vertical accelerations are corrected with the Eótvös and the free-air correction, and the theoretical gravity is removed. Note the high power in the signal at the Nyquist frequency $(0.5 \mathrm{~Hz})$. (b) The filter response of the 300-s Gaussian and the $6 \times 20$-s RC filters shown from dc to Nyquist indicate the excellent high-frequency attenuation of the $\mathrm{RC}$ filter and the poor high-frequency attenuation of the Gaussian. 
The geologic wavelength represents the common definition of anomaly wavelength used to define anomaly resolution, equivalent to the half-wavelength of a sinusoid. We define an additional measure of the wavelength that maps the geologic wavelength into the Fourier domain. In the Appendix, we demonstrate that the sinusoid with a wavelength twice the geologic wavelength provides a good frequency-domain approximation to the gravity anomaly, which we term the "Fourier" wavelength:

$$
\lambda_{f}=2 \lambda_{g}=3.1 z_{c}
$$

The Fourier wavelength $\lambda_{f}$ defines the detection threshold, the estimate of the upper bound of the gravity waveband, that can be converted to frequency with the aircraft speed. We modify this theory to relate these anomaly wavelengths to the distance to the top of the sphere $z$, because this is the only information available in an actual survey (see Appendix).

This method for identifying the signal waveband is illustrated by data from the West Antarctic survey (Figure 2). Given an average survey elevation of $2500-3000 \mathrm{~m}$ above bedrock and an expected density contrast of $1.2 \times 10^{3} \mathrm{~kg} / \mathrm{m}^{3}$ for the rock/ice interface, we estimate that the gravity waveband should extend

a)

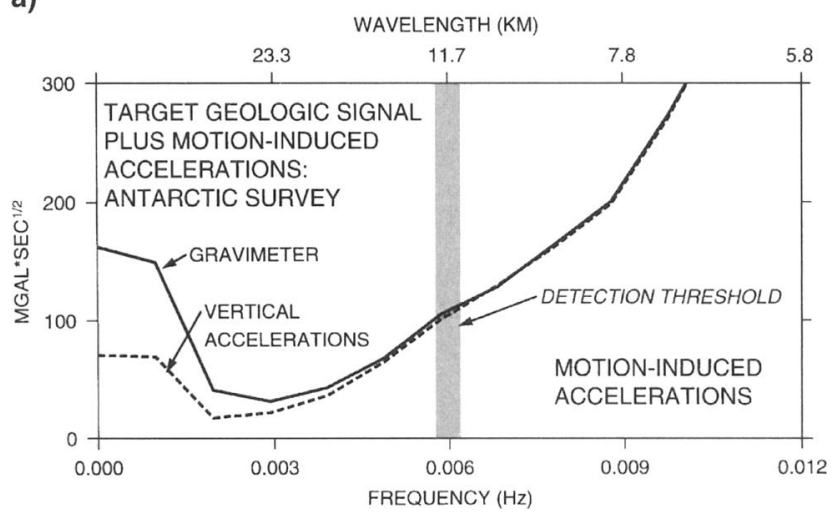

b)

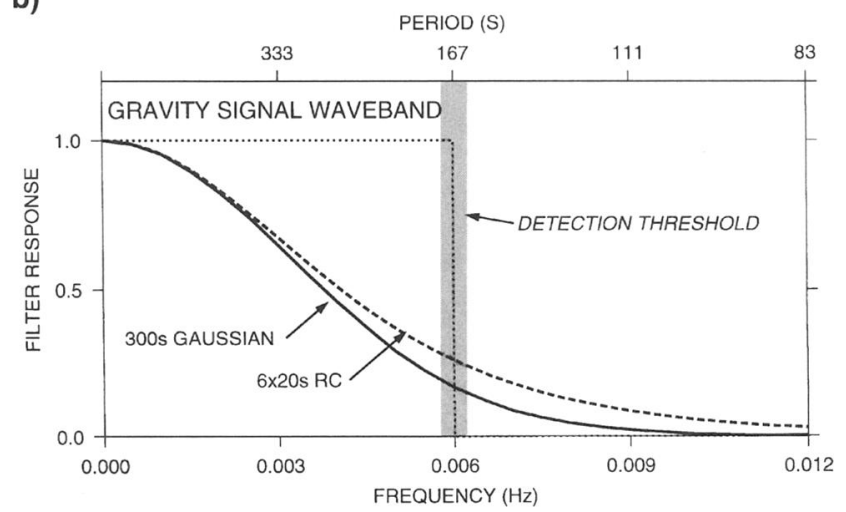

FIG. 2. (a) Amplitude spectrum of gravimeter measurement versus vertical accelerations averaged for 33 flights, shown in the lowest $2 \%$ of the spectral range. The difference in these two signals is the spectrum of the free-air anomaly. The detection threshold indicates the effective upper limit of the signal waveband. Note that the two series converge near the detection threshold. (b) The filter responses of the 300-s Gaussian and the $6 \times 20$-s RC filters illustrate the significant attenuation of the signal waveband. from dc to $0.0058-0.0067 \mathrm{~Hz}$ based upon a Fourier wavelength between 10.4 and $12 \mathrm{~km}$ and a $70 \mathrm{~m} / \mathrm{s}$ aircraft velocity. The corresponding geologic wavelength is 5.2 to $6 \mathrm{~km}$. Averaged spectral estimates of the gravimeter measurement and the aircraft vertical accelerations combined with the Eötvös, free-air, and latitude corrections are shown for 33 survey lines. The difference of these spectra is the spectrum of the free-air anomaly. The difference between the gravimeter's response and the calculated corrections is largest at low frequencies and approaches zero at the detection threshold. These spectral estimates illustrate the dominance of the long-wavelength geologic signals in the gravity waveband and the signal drop-off near $0.006 \mathrm{~Hz}$.

\section{Noise in the data}

Airborne gravity is acquired in a dynamic environment that introduces substantial noise into the measurement. The aggressive low-pass filtering necessary to reduce the effects of this noise can also attenuate part of the signal waveband. The extreme attenuation of the low-pass filter becomes the controlling factor on the resolution of the data.

Noise in the free-air anomaly is attributed to uncorrected motion-induced error in the gravimeter measurement and errors in the interferometric GPS positions. Most noise in the gravimeter reading scales with the amplitude of aircraft motion, as has been shown by the correlation between gravimeter error and sea state in marine gravimetry (LaCoste, 1967). Aircraft vertical acceleration for the West Antarctic data is at a minimum at $0.002 \mathrm{~Hz}$, then increases with increasing frequency to completely dominate the data above $0.006 \mathrm{~Hz}$ (Figure 2). Horizontal accelerations induced by course corrections and air turbulence also grow with increasing frequency and cause offlevel error. Offlevel corrections only compensate for a portion of the total error introduced.

The quality of the interferometric position from GPS carrier phase data is a function of the number of satellites visible, the geometry of their positions in the sky, the baseline distance between the aircraft and the base station, and the multipath environment of the GPS antenna mounted atop the aircraft fuselage. Static tests indicate that some noise exists at the longest wavelengths in the GPS vertical acceleration data $(2-3 \mathrm{mGal}$ at dc to $0.0083 \mathrm{~Hz}$ ) (Peyton, 1990), and additional noise that results from multipath in the airborne environment has not been quantified. At high latitudes, the quality of the solution is further degraded by increased ionospheric interference and low satellite elevation.

\section{Traditional filters}

Two low-pass filters employed in marine gravimetry have been used in airborne gravimetry. These filters are the $6 \times 20$-s RC filter and the Gaussian filter. The $6 \times 20$-s RC filter has been widely used in conjunction with the LaCoste and Romberg (L\&R) S marine meter. The output of the L\&R S meter is internally filtered with three stages of 20 -s RC filtering, and, without equipment modification, an unfiltered output is not available. Increasing numbers of $L \& R$ meter users have modified their meters to output unfiltered measurements enabling other filters to be implemented.

The RC filter is a recursive infinite impulse response (IIR) filter that feeds the output of the filter back into the input to 
generate future output. This feedback mechanism creates a phase lag in the meter output that must be removed by applying three additional stages of 20 -s RC filtering with time reversed. Thus the filtered output of this meter mandates the use of the $6 \times 20$-s RC filtering scheme. The $6 \times 20$-s RC filter well attenuates the high frequency components (Figure 1b), but also heavily attenuates even the lowest frequency components except dc (Figure 2b).

Another commonly used filter in marine gravimetry with the Bell Aerospace BGM-3 gravimeter is the Gaussian filter. The BGM-3 meter internally filters the output with a filter length of about $4 \mathrm{~s}$, much less severe than the $3 \times 20$-s RC. BGM-3 users have used Gaussian filters because of the ease of use and the ability to adjust the amount of filtering by specifying the filter's $6 \sigma(6 \times$ standard deviation $)$ width. The Gaussian filter is a finite impulse response (FIR) filter that, by definition, has inherently good phase and stability characteristics. Data lost to the filtering process is equal to the operator length. At a $6 \sigma$ width of $300 \mathrm{~s}$, the response of the Gaussian filter approximates the $6 \times 20$-s RC filter at the lowest frequencies (Figure 2). The Gaussian's filter response, which is also a Gaussian function, illustrates that no pass band exists where the signal is unattenuated by the filter because all frequencies except dc are attenuated to some degree. The 300 -s Gaussian filter has significantly poorer high-frequency attenuation than the $\mathrm{RC}$ filter as a result of truncating the time domain operator to the $6 \sigma$ width. The high-frequency attenuation of only four orders of magnitude is certainly inadequate to suppress the six to seven orders of magnitude of high-frequency noise in the data (Figure 1). An additional short filter is often used to improve high-frequency attenuation.

These traditional filters are more appropriate for marine gravimetry than for airborne gravimetry because the slower marine survey speeds with respect to the anomaly wavelengths shrink the signal waveband to a much narrower region of the spectrum just slightly above dc. Attenuation of the signal near dc by these filters is slight. With the signal waveband identified for the West Antarctic survey (Figure 2a), the substantial attenuation of the target gravity signal in the airborne data by the traditional filters can be seen (Figure 2b).

\section{Optimizing filter performance: Filter selection and design}

The ideal filter for airborne data would have controllable frequency roll-off characteristics so that a passband and transition zone could be easily specified, with adequate attenuation of the high-frequency noise in the stop band. A variety of filters exist that potentially could satisfy these requirements. Unfortunately, airborne data present a difficult challenge for low-pass filters. Low-pass filters must remove the top $99 \%$ of the frequency range of the data. The very low frequency cutoffs and steep roll offs required plus the high-amplitude nature of the noise often cause the introduction of undesirable filtering artifacts that can result in significant data loss. IIR filters (e.g. Butterworth, Chebyshev, elliptic) on airborne data introduce initial transients of $400-800 \mathrm{~s}$, even when the data are detrended and windowed prior to filtering (Childers, 1996). FIR filters (using a Parks-McClellan algorithm or various windowing techniques) are only able to attenuate the uppermost $80 \%$ of the frequency range in a single pass. FIR filters and the complex operations required to minimize artifacts including iterative filtering, decimation, and data mirroring result in unacceptable data loss for the short West Antarctic survey lines (1500$2000 \mathrm{~s}$ ) (Childers, 1996). These filters might be appropriate where recording is continuous throughout a survey, or where 10-12 minutes of data loss along the line can be tolerated.

In this study, we use a filter that operates directly in the frequency domain, which we term the fast Fourier transform (FFT) filter. The time series to be filtered is Fourier transformed, and a cosine taper is applied to the Fourier coefficients, which are then inverse transformed back to the time domain. In tests with both simulated and actual airborne data, this filter performs reliably with only about $100 \mathrm{~s}$ of data corruption at each end of a filtered series (Childers, 1996).

The following sequence of steps are executed to stabilize the transform and to minimize the Gibbs phenomenon or "ringing" at the ends of the time series during the filtering process: (1) the time series has its mean and trend removed, and the ends of the series are "windowed" with a cosine taper over $50 \mathrm{~s}$; (2) the time series is padded with approximately three times as many zeros as data points (to the nearest power of two); (3) the time series is transformed into the frequency domain with the FFT; (4) the Fourier coefficients of the series are multiplied by a filter function (here, a cosine taper); (5) the coefficients are inverse transformed to the time domain; and (6) the mean and trend are restored. The cosine taper filter function equals 1 in the passband, 0 in the stop band, and the transition zone is formed by a cosine function one-half wavelength long. The transition zone is specified by the width of the cosine taper and by the frequency where attenuation begins. The filter function is mirrored so as to be applied to both positive and negative frequencies alike.

An iterative process is necessary to determine the optimum filter design for a given survey. The first stage is to determine the detection threshold for the survey based upon the survey parameters of aircraft altitude and speed, distance to source $z$, and expected density contrast, using the method in the Appendix. Then, the Fourier wavelength can be determined from equation (2). The Fourier wavelength can then be converted to the detection threshold frequency by aircraft speed. The second stage is to design a "desired" filter with its half-power point at the detection threshold. This filter would pass the gravity signal unattenuated. The third stage is to evaluate how well this filter design attenuates the noise and preserves the signal. Generally, the measurement noise will demand more attenuation than this desired filter provides. The final stage of the filter design process is to gradually lower the frequency cutoff of the filter and experiment with different cosine widths until the noise is optimally attenuated. This iterative process is facilitated by having the low-pass filtering be the final step in the processing strategy.

\section{FILTER EVALUATION WITH AIRBORNE DATA}

\section{The West Antarctic survey}

The first survey data we evaluate in this study were collected during the 1991-1992 field season of an aerogeophysical survey over the West Antarctic ice sheet (Blankenship et al., 1993). For this survey, a Bell Aerospace BGM-3 gravimeter, an ice-penetrating radar, a scalar magnetometer, and laser and pressure altimeters were installed in a de Havilland Twin 
Otter aircraft. Real-time navigation instrumentation included a Litton 92 inertial navigation system, a radio navigation system, and a Trimble 2000 GPS receiver. Ashtech P-12 GPS receivers recorded carrier phase observables for precise postsurvey positioning. The West Antarctic data were acquired at altitudes above bedrock ranging from 2500 to $3300 \mathrm{~m}$ (due to the thick ice cover of 1000 to $3000 \mathrm{~m}$ ) and at ground speeds of approximately $135 \mathrm{kn}$ (about $70 \mathrm{~m} / \mathrm{s}$ ). Survey lines were short, typically $140 \mathrm{~km}$ long, and were flown in 1500-2100 s. During one month of surveying, more than 25000 survey line-km were acquired. The $220 \times 220 \mathrm{~km}$ region was covered with a grid of orthogonal lines spaced $5.3 \mathrm{~km}$ a part.

GPS carrier phase measurements were collected at a $1-\mathrm{Hz}$ data rate and recorded simultaneously by three Ashtech receivers in the aircraft and three receivers at a centrally located base station. The GPS data were processed and the precise aircraft position solutions calculated with XOMNI software (Ball et al., 1995), a modified version of the OMNI software developed by Dr. Gerald Mader (Mader, 1986).

The Bell Aerospace BGM-3 marine gravimeter, modified for the airborne environment, consists of a Bell model XI inertialgrade accelerometer mounted on a two-axis, gyro-stabilized platform that maintains the alignment of the sensitive axis of the accelerometer with the time-averaged apparent local vertical. Because offleveling error from horizontal accelerations decreases with increasing platform period (LaCoste, 1967), the natural period of the platform of this meter was lengthened from 66 to $666 \mathrm{~s}$ to compensate for the dynamic motion of the aircraft.

The West Antarctic survey contains a line that was surveyed on two separate occasions. In the absence of ground truth data, reflights of the same line provide our best opportunity to evaluate which filter design maximizes the repeatability of the airborne data. More specifically, this analysis allows us to determine what degree of filtering is required to remove most of the noise in the gravity waveband.

The detection threshold is approximately $12 \mathrm{~km}$ or about $0.006 \mathrm{~Hz}$ based upon the survey parameters. The "desired" filter, FFT filter 1 (Figure 3), has its half-amplitude point at the detection threshold. Unfortunately, noise dominates the signal at the upper end of the signal waveband, requiring a modification of the filter so the maximum attenuation is reached at $0.006 \mathrm{~Hz}$ (FFT filter 2, Figure 3).

We filter the repeat lines with the two FFT filters, a traditional $6 \times 20$-s RC filter, and a 300-s Gaussian filter augmented with an additional 20-s Gaussian to improve high-frequency attenuation (Figure 3). Our desired FFT filter 1 passes a considerable component of noise, reflected in the $5.88-\mathrm{mGal} \mathrm{rms}$ difference between reflights. The increased attenuation of the optimum FFT filter 2 eliminates the noise above $0.006 \mathrm{~Hz}$ while preserving the lowest frequencies unattenuated. The rms difference between reflights for the FFT filter 2 is slightly better than for the RC and Gaussian filters; however, the more significant improvement is in the amplitude enhancement of the long wavelength anomalies. For flight A, the FFT filter 2 increases the peak-to-trough amplitude by $8 \mathrm{mGal}$ over the Gaussian filtered version in the region of 15 to $35 \mathrm{~km}$ along track. No clear enhancement in anomaly amplitude is observed in flight A due to the offlevel error between 25 and $35 \mathrm{~km}$.

\section{NRL East Pacific ground truth comparison}

The second survey evaluated here was conducted by the Naval Research Laboratory (NRL) in the spring of 1997. Six lines were flown over seamounts off the coast of California previously surveyed with classic marine gravity techniques by the Naval Oceanographic Office (NavOceanO). This survey provides a unique opportunity to compare airborne data to highquality marine data, and provides an excellent test of whether modifying the filter design impacts data accuracy. The survey was flown aboard the Navy's P-3A Orion aircraft at close to minimum practical altitude $(300 \mathrm{~m})$ and speed $(150 \mathrm{kn})$ to aim for the highest resolution attainable from this aircraft. Survey lines were about $300 \mathrm{~km}$ long. The overlap with the marine profiles was only $140 \mathrm{~km}$. Real time navigation was provided by a Litton inertial system. An Ashtech Z-12 GPS receiver on board the aircraft and another at a base station measured the carrier phase data that were postprocessed with XOMNI

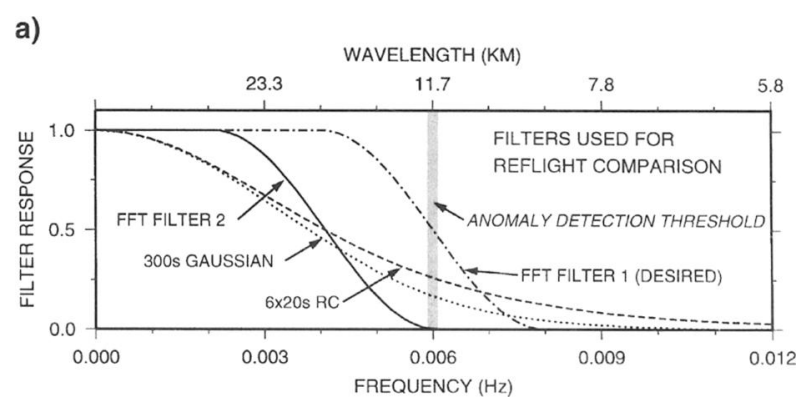

b)
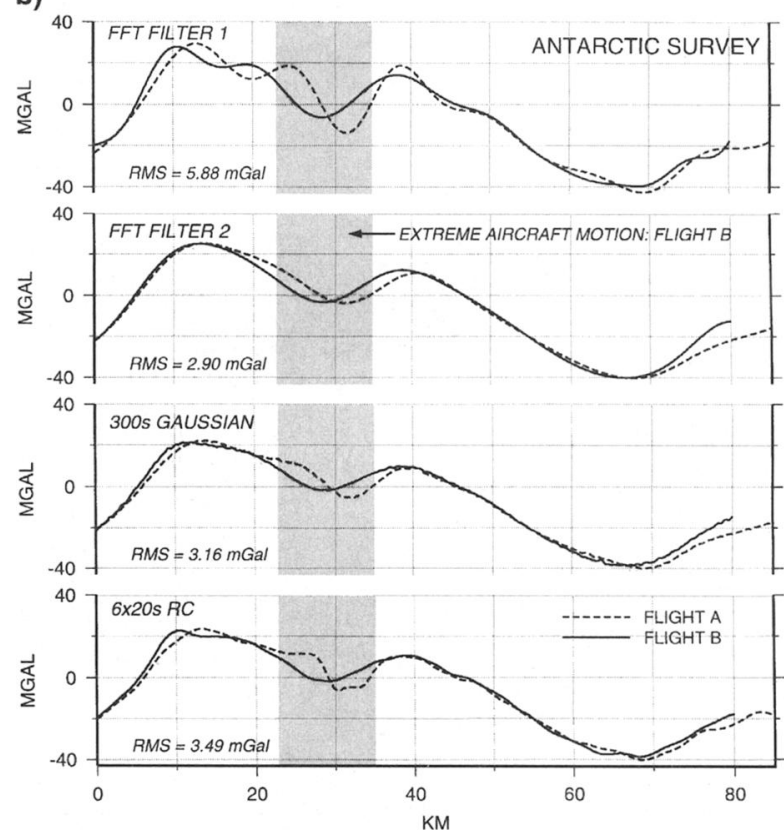

FIG. 3. Reflight analysis of the West Antarctic data. (a) Filter responses for the FFT filters, the 300-s + 20-s Gaussian, and the $6 \times 20$-s RC filters used in the reflight analysis. (b) Two independent measurements of the same survey line processed with the various filters. The shading indicates a portion of flight $B$ affected by extreme aircraft motion. 
software for precise aircraft positioning and vertical acceleration determination. Additional altimetry data were provided by a radar altimeter. The gravimeter was a L\&R S marine meter upgraded by the ZLS Corporation to provide digital platform control and variable platform period.

Estimating the gravity signal waveband requires evaluation of the local bathymetry. Regional water depth ranges from 3800 to $4000 \mathrm{~m}$ with superimposed seamounts that extend to within $600 \mathrm{~m}$ of the surface. The large seamounts dominate the gravity signal and provide substantial power at relatively short wavelengths. Given an aircraft altitude of $300 \mathrm{~m}$ and a density contrast between the seamount and water of $1.65 \times 10^{3} \mathrm{~kg} / \mathrm{m}^{3}$, the smallest anomaly observable at $900 \mathrm{~m}$ from the edge of the source has a geologic wavelength $\lambda_{\mathrm{g}}$ of $1.95 \mathrm{~km}$ and a Fourier wavelength $\lambda_{f}$ of $3.9 \mathrm{~km}$. At aircraft survey speeds of $150 \mathrm{kn}$ $(75.6 \mathrm{~m} / \mathrm{s}), \lambda_{f}$ corresponds to a period of $52 \mathrm{~s}$ and a detection threshold frequency of $0.019 \mathrm{~Hz}$. This frequency specifies a filter cutoff much higher than the measurement noise would permit. Another approach is to approximate the seamount as a sphere with radius one-half the seamount height. $\mathrm{A}$ radius of $R=(4000-600) / 2=1700 \mathrm{~m}$ specifies a $\lambda_{g}$ of $4.49 \mathrm{~km}$ and a $\lambda_{f}$ of $7.97 \mathrm{~km}$, and predicts a detection threshold frequency of $0.0095 \mathrm{~Hz}$. Regardless of which of these frequencies we select to be the detection threshold, it is clear that we expect substantial power at wavelengths shorter than anticipated for the West Antarctic survey.

NRL traditionally has reduced the P-3 airborne data using the $6 \times 20$-s RC filter with a short FIR filter to slightly sharpen the frequency roll off (Brozena and Peters, 1988) (Figure 4a). The FFT filter that minimizes the rms difference with the upward-continued marine data begins its frequency roll off at 0.003 , has its half-amplitude point at 0.005 , and reaches maximum attenuation by $0.007 \mathrm{~Hz}$ (Figure 4a). Comparison of three of the airborne survey lines with the marine data demonstrates that maximum amplitudes of the airborne anomalies are increased by as much as $20 \mathrm{mGal}$ when the FFT filter is used instead of the RC filtering scheme (Figure 4b). The average rms differences between the airborne and upward-continued shipboard data for the three lines by filter type are: $\mathrm{RC}=3.99 \mathrm{mGal}$, 300 -s Gaussian $=3.50 \mathrm{mGal}$, FFT $=2.04 \mathrm{mGal}$ (a $49 \% \mathrm{im}$ provement over the RC filter). Rms differences for the individual lines are listed in Table 1.

The optimum FFT filter, with its half-amplitude point at $0.0045 \mathrm{~Hz}$, has a much lower cutoff than the detection threshold frequency of $0.0095 \mathrm{~Hz}$. Attempts to pass more of the signal waveband using filters with higher cutoffs retained unacceptable levels of noise in the free-air anomaly. This need to attenuate the upper half of the gravity waveband indicates that measurement noise tends to dominate the geologic signal in this portion of the waveband.

Table 1. Differences between airborne and upward-continued marine gravity data (in rms $\mathbf{m G a l}$ ) for each East Pacific survey line shown in Figure 4 for the three lowpass filters employed.

\begin{tabular}{lccc}
\hline & $6 \times 20$-s RC filter & 300-s Gaussian & FFT filter \\
\hline Line 1 & 5.27 & 4.62 & 1.89 \\
Line 2 & 2.13 & 1.93 & 1.86 \\
Line 3 & 4.58 & 3.95 & 2.37 \\
\hline
\end{tabular}

\section{DISCUSSION}

The goal of this filter study was to determine if the accuracy and resolution of airborne gravity could be improved by careful filtering of the data. By tailoring the filter to the survey, we were able to enhance the anomaly amplitudes for both surveys, improving the rms difference slightly for the Antarctic reflight data and significantly for the East Pacific ground truth comparison. The most dramatic improvement was demonstrated for the East Pacific survey because the noise characteristics of the data allowed the passband to be increased, and because there is substantial power in the gravity signal in the increased passband of the FFT filter. The results of these two studies show that filters can be carefully designed for specific survey parameters to improve anomaly recovery.

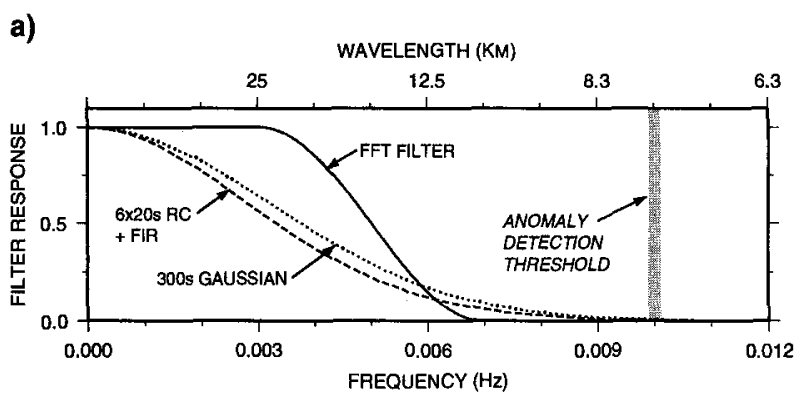

b)
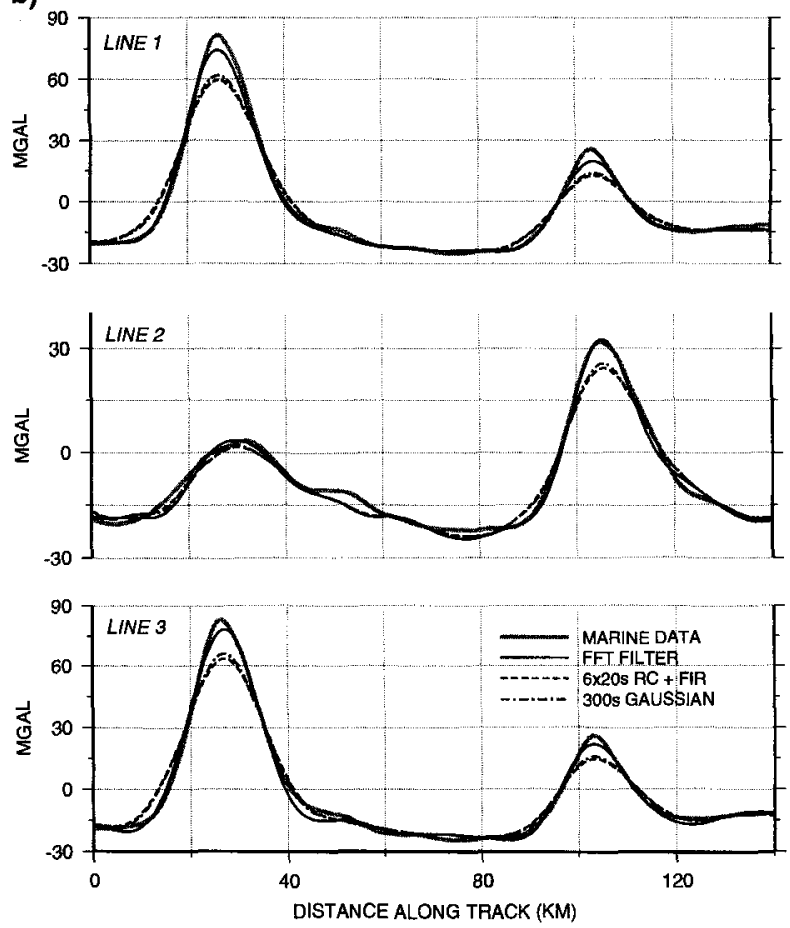

FIG. 4. Comparison of East Pacific airborne data with upward continued shipboard data. (a) The filter response for filters used in the comparison: the six stages of 20 -s $(6 \times 20$-s) RC plus a small FIR filter to slightly sharpen the attenuation, the 300-s +20 -s Gaussian, and the best performing FFT filter. The detection threshold corresponds to the signal waveband of the seamounts in the survey. (b) Airborne data filtered with the three filters are compared to shipboard data for three profiles. The rms difference between the filtered airborne and the upward continued marine data for each line is listed in Table 1. 
While improving wavelength and amplitude recovery, filters with passbands and steep frequency roll offs can also introduce errors that could be mistaken for gravity anomalies. These errors are expressed as oscillations in the free-air anomaly before and after impulselike anomalies such as seamounts, as evident by the anomaly lows either side of the seamounts in Line 3 (Figure 4). A similar effect would be seen at abrupt steps in the free-air anomaly. Such filter-induced artifacts from steep roll-off filters pose a concern in other geophysical data as well, where precursory filter artifacts in earthquake seismic data can be mistaken for nucleation phases (Scherbaum and Bouin, 1997).

The range of filter-induced anomaly distortion is demonstrated by applying the various filters to the smallest predicted anomaly for the East Pacific survey (Figure 5). Filters without passbands, such as the RC filter or the Gaussian, tend to broaden the anomaly wavelength and diminish the amplitude, but do not introduce oscillations. Filters with passbands and steeper frequency roll offs, such as the optimum and desired FFT filters, improve anomaly amplitude and wavelength recovery, but introduce negative and positive "lobes." The nature and amplitude of the lobe distortion can be controlled to some

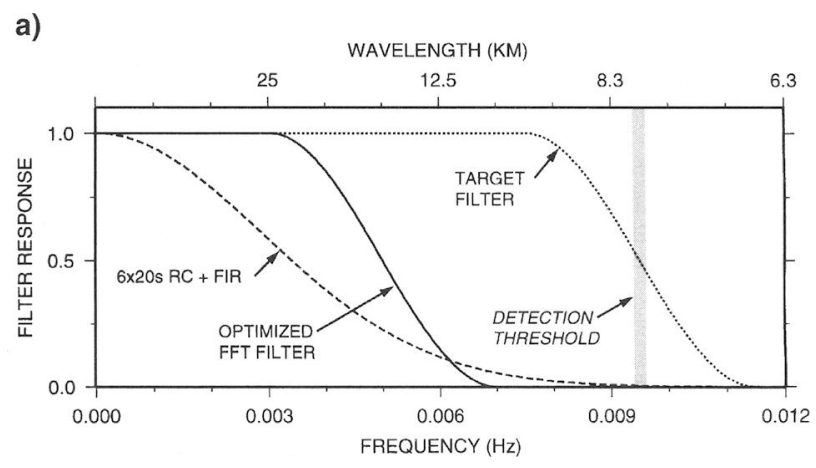

b)

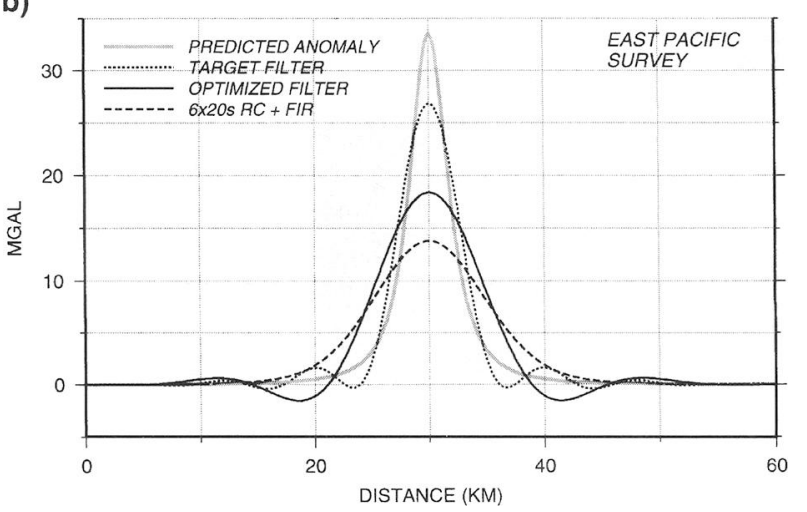

FIG. 5. Anomaly distortion by the low-pass filter is illustrated. (a) Filter responses for the $6 \times 20$-s RC filter and FFT filter from Figure 4 are shown. The desired filter has its half-amplitude point at the detection threshold. (b) The smallest detectable anomaly for the East Pacific survey is filtered using the filters shown in (a). The RC filter broadens and attenuates the anomaly, the FFT filter preserves slightly more amplitude but introduces negative "lobes" either side of the peak. The desired filter better preserves the amplitude and wavelength of the smallest anomaly, but cannot be used because it passes too much noise. For clarity, the 300-s Gaussian is not displayed because of its similarity to the RC filter. degree by the filter design. Lobes are minimized with gentle frequency roll offs and are removed by eliminating the passband.

Some distortion of the gravity anomalies by the low-pass filtering is unavoidable because of the low-frequency cutoffs required to minimize the noise in the data. Acceptable anomaly distortion may vary according to survey objectives. In some cases, it may be more acceptable to have some peak amplitude attenuation and broadening in exchange for no lobe distortion, whereas other surveys may demand better amplitude and wavelength recovery at the expense of some lobe distortion. The advantage to using the FFT filter we describe in this study is that these various filter designs are easily crafted and implemented.

An additional advantage to the FFT filter is that any functional form can be used in the filter function to describe the filter's frequency roll off. A filter with a Gaussian function for a taper but no passband is simply a frequency domain implementation of the Gaussian filter. This frequency-domain Gaussian filter has several characteristics that make it a viable option for noisy airborne data. First, this filter has excellent highfrequency attenuation that is unattainable with a time-domain operator truncated to the $6 \sigma$ width. Secondly, because data loss to the transform is roughly constant regardless of the filter employed, very long Gaussian filters can be implemented without the additional data loss of the time-domain filter.

One difficulty with the FFT filter occurs when large outliers exist at the beginning or end of a series that may be the result of extreme aircraft motion associated with a turn, for example. It is best to trim off these outliers prior to filtering to prevent a large oscillation from propagating into the series.

The fundamental assumption of our study is that if corrections for aircraft motion are properly calculated and removed from the airborne data, then a filter that passes more of the signal above the detection threshold will provide an improved recovery of the target geologic signal. The remaining rms misfits for both surveys clearly indicate that substantial noise remains in the gravity waveband even after heavy filtering. The presence of this noise in the signal waveband means that lowpass filtering is not enough in itself to remove this noise from the data. The motion-induced noise in the signal waveband remains an unresolved problem in airborne gravimetry.

\section{CONCLUSIONS}

Low-pass filtering plays an important role in determining the amplitude and spectral content of the airborne-measured free-air gravity anomaly. In this study, we evaluate the choice and design of the low-pass filter used in data processing. We note that the traditional $6 \times 20$-s RC and 300-s Gaussian filters inherited from marine gravimetry attenuate a significant portion of the waveband of the target geologic signal as measured from aircraft. We develop a new approach to low-pass filtering that involves identifying the waveband of the gravity signal based upon survey parameters and an iterative approach to filter design where the design is repeatedly tested and modified to yield optimum results.

We apply our new technique to aerogravimetry data from two surveys. A reflight analysis of West Antarctic data shows that this technique yields an rms improvement of $0.25 \mathrm{mGal}$ and an 8-mGal increase in peak anomaly amplitude. A "ground truth" comparison between airborne and marine gravimetry 
measurements over seamounts in the eastern Pacific Ocean demonstrates an $49 \%$ improvement of the rms correspondence $(1.95 \mathrm{mGal})$ and as much as a 20 -mGal improvement in anomaly amplitudes with the use of the new technique. These results indicate that filters tailored to the specific survey that pass more of the gravity signal band unattenuated can improve the recovery of the amplitude and wavelength of gravity anomalies measured from the airborne platform.

\section{ACKNOWLEDGMENTS}

This work was supported through NSF Office of Polar Programs grants OPP-9100155, OPP-9120638, and OPP-9319854, NASA Global Change Fellowship NGT30166, and the Office of Naval Research, P.E. No. 0601153N. Additional funding for aircraft support for the East Pacific survey was provided by CNMOC (Commander Naval Meterological and Oceanographic Command) and NIMA (National Imagery and Mapping Agency). The authors thank Alan Herring and Richard Hansen for their thoughtful reviews. Thanks also to the people who contributed to this work in many ways: Bob Arko, David Ball, Chris Small, Doug Martinson, and Enders Robinson, who contributed ideas on refining the FFT filter.

\section{REFERENCES}

Ball, D. G., Jarvis, J. L., Brozena, J. M., and Peters, M. F., 1995, XOMNI user and technical documentation: Naval Research Laboratory Report NRL/MR/7420-95-7774.

Blankenship, D. D., Bell, R. E., Hodge, S. M., Brozena, J. M., Behrendt, J. C., and Finn, C. A., 1993, Active volcanism beneath the west Antarctic ice sheet and implications for ice sheet stability: Nature, 361, 526-529.
Brozena, J. M., 1994, CASERTZ 91-92: Kinematic GPS, airborne gravity and surface topography (abstract): EOS, 75, 102 .

Brozena, J., Chalona, M., Forsberg, R., and Mader, G., 1992, The Greenland aerogeophysics project (abstract): EOS, 73, 130.

Brozena, J. M., and Peters, M. F., 1988, An airborne gravity study of eastern North Carolina: Geophysics, 53, 245-253.

Childers, V. A., 1996, Gravimetry as a geophysical tool: airborne gravimetry and studies of lithospheric flexure and faulting: Ph.D. thesis, Columbia Univ.

Childers, V. A., Peters, M. F., and Brozena, J. M., 1997, Error analysis of the NRL airborne gravimetry system: Internat. Symp. on Kinematic Systems in Geodesy, Geomatics, and Navigation, 625-632.

Gumert, W. R., 1995, Third generation aerogravity system: Internat. Symp. on Kinematic Systems in Geodesy, Geomatics, and Navigation, $153-162$.

Harlan, R. B., 1968, Eötvös corrections for airborne gravimetry: J. Geophys. Res., 73, $4675-4679$.

Harrison, J. C., MacQueen, J. D., Rauhut, A. C., and Cruz, J. Y., 1995 The LCT airborne gravity system: Internat. Symp. on Kinematic Systems in Geodesy, Geomatics, and Navigation, 163-169.

LaCoste, L. J. B., 1967, Measurement of gravity at sea and in the air: Rev. Geophys., 5, 477-526.

Mader, G. L., 1986, Dynamic positioning using GPS carrier phase measurements: Manuscr. Geod., 11, 272-277.

Olesen, A. V., and Forsberg, R., 1997, Airborne gravimetry using the LaCoste \& Romberg gravimeter-An error analysis: Internat. Symp. on Kinematic Systems in Geodesy, Geomatics, and Navigation, 613617.

Peters, M. F., and Brozena, J. M., 1995, Methods to improve existing shipboard gravimeters for airborne gravimetry: Internat. Symp. on Kinematic Systems in Geodesy, Geomatics, and Navigation, 39 46.

Peyton, D. R., 1990, An investigation into acceleration determination for airborne gravimetry using the Global Positioning System: Univ. of New Brunswick Technical Report 149.

Scherbaum, F., and Bouin, M. P., 1997, FIR filter effects and nucleation phases: Geophy. J. Internat., 130, 661-668.

Telford, W. M., Geldart, L. P., and Sheriff, R. E., 1990, Applied geophysics: Cambridge Univ. Press.

Turcotte, D. L., and Schubert, G., 1982, Geodynamics: John Wiley and Sons.

\section{APPENDIX}

\section{DETERMINING THE SMALLEST OBSERVABLE ANOMALY}

We estimate the upper limit of the gravity waveband by determining the wavelength of the smallest point source anomaly observable from the aircraft altitude. The gravitational attraction of a sphere is the same as if all the mass were at its center, essentially that of a point source. The gravity effect of a sphere at a point $P$ (Figure A-1a) directed along $r$ is

$$
g_{r}=\frac{\gamma d m}{r^{2}}
$$

The vertical component $g_{z}$ is

$$
g_{z}=g_{r} \cos \theta=\frac{\gamma d m z_{c}}{r^{3}},
$$

where $\gamma$ is the universal gravitational constant and $z_{c}$ is the aircraft height above the sphere's center. The excess mass $d m$ represented by the sphere is $4 \pi R^{3} \sigma / 3$, where $R$ is the radius of the sphere and $\sigma$ is the density contrast of the sphere with its surroundings. $r^{2}$ can be expressed as $x^{2}+z_{c}^{2}$. Thus, equation (A-2) becomes

$$
g_{z}=\frac{4 \gamma \pi R^{3} \sigma z_{c}}{3 r^{3}}=\frac{4 \gamma \pi R^{3} \sigma}{3} \cdot \frac{z_{c}}{\left(x^{2}+z_{c}^{2}\right)^{3 / 2}}
$$

Equation (A-3) can be rearranged by factoring out $z_{c} / z_{c}^{3}$ from the right-hand fraction:

$$
g_{z}=\frac{4 \gamma \pi R^{3} \sigma}{3 z_{c}^{2}} \cdot \frac{1}{\left(1+x^{2} / z_{c}^{2}\right)^{3 / 2}} .
$$

The right-hand fraction of equation (A-4) describes the shape of the gravity anomaly and is a function only of the height $z_{c}$ above the center of the sphere. The width of the anomaly is related to $z_{c}$ by the expression

$$
z_{c}=1.3 w_{1 / 2}
$$

where $w_{1 / 2}$ is the half-width of the anomaly at the halfamplitude point (Telford et al., 1990). To apply this theory, however, we need to relate the width of the anomaly to the height $z$ above the top of the sphere, because in real circumstances this is the only information we have. The problem assumes greater complexity, for now we need to impose geologically reasonable values and draw some conclusions about the size of the sphere so that the distance $z_{c}$ to its center can be inferred.

To be discernible above the noise inherent in the measurement, the buried sphere must generate at least a $2-\mathrm{mGal}$ anomaly. The left-hand fraction of equation (A-4) defines $g_{\text {max }}$, the amplitude of the anomaly maximum. Given a specified density contrast of the sphere with its surroundings, the 
a)

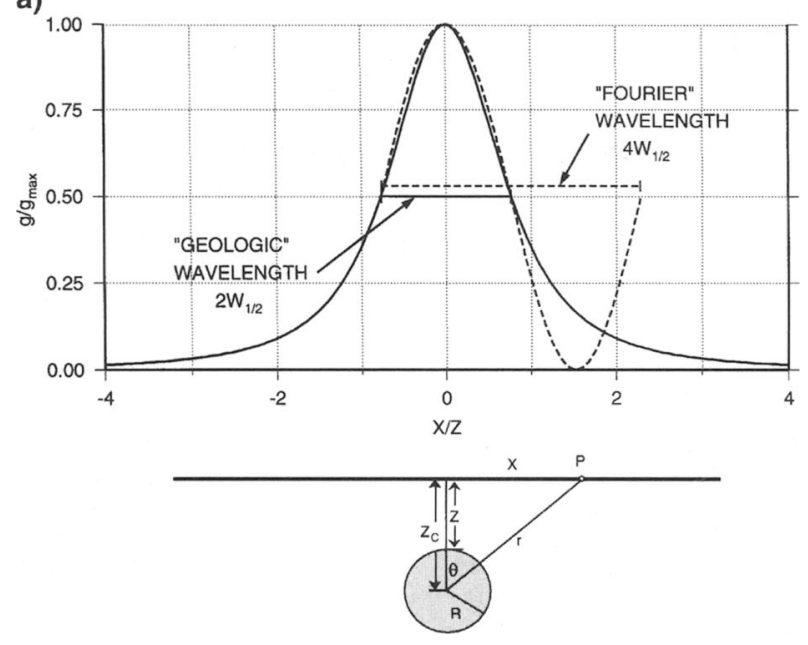

b)

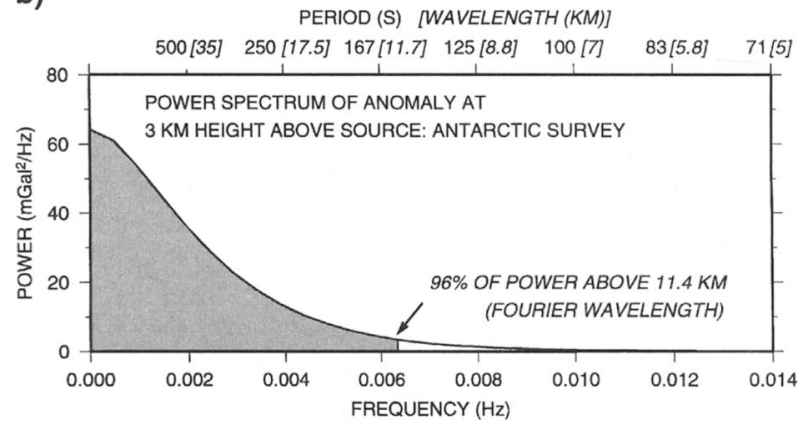

FIG. A-1. (a) The gravity anomaly generated by a buried sphere is illustrated by the solid line. Its "geologic" wavelength is characterized by twice the "half-width" $w_{1 / 2}$, defined as half the width of the anomaly at half the maximum amplitude. The anomaly wavelength can be approximated in the frequency domain (Fourier wavelength) by a sinusoid of wavelength $4 w_{1 / 2}$ (dashed line). (b) A total of $96 \%$ of the power in the signal of the smallest observable anomaly for a $3-\mathrm{km}$ altitude is contained in the signal from dc to the frequency corresponding to the Fourier wavelength. This wavelength can be used to estimate the upper limit of the gravity signal waveband.

measurement height $z$ above the top of the sphere, and a minimum gravity anomaly $\left(g_{\max }=2 \mathrm{mGal}\right)$, we can solve the $g_{\max }$ expression for the radius $R$ of the sphere. The expression for $g_{\text {max }}$ is nonlinear in $R^{3}$ and $z_{c}^{2}$, and cannot be solved directly for $R$. But $z_{c}$ can be expressed as a function of $R$, so we make a change of variable and rearrange the expression for $g_{\max }$. We introduce the variable $z$ to represent the distance from the aircraft to the top of the buried sphere (Figure A-1a), so that $z_{c}=z+R$, and solve for $\sigma$ :

$$
\sigma=\frac{3 g_{\max }}{4 \gamma \pi} \cdot \frac{(z+R)^{2}}{R^{3}} .
$$

Using values for $g_{\max }=2 \mathrm{mGal}, \gamma=6.67 \times 10^{-11} \mathrm{~m}^{3} /\left(\mathrm{kg} \mathrm{s}^{2}\right)$, equation (A-6) becomes

$$
\sigma=71584 \cdot \frac{(z+R)^{2}}{R^{3}}
$$

Selecting $z$ to reflect aircraft altitudes, we iteratively estimate values of $R$ until the desired value of the density contrast $\sigma$ is obtained. $z_{c}$ is then determined from $z+R$, and inserted into equation (A-5) to determine the half-width $w_{1 / 2}$.

The "geologic" wavelength of the smallest anomaly (Figure A-1a) is defined as twice the half-width:

$$
\lambda_{g}=2 w_{1 / 2}=1.54 z_{c} .
$$

To define the gravity signal waveband in terms of frequency, we must map this geologic wavelength into the frequency domain. We note that the sinusoid with a wavelength twice the geologic wavelength, or $4 w_{1 / 2}$, nearly approximates the anomaly with its half-wavelength (Figure A-1a). The power spectrum of the smallest anomaly observable for 3-km aircraft altitude (for the West Antarctic survey) illustrates that there is power in the signal across a fairly wide band. However, $96 \%$ of the power in the signal is contained in the waveband from dc to $0.0062 \mathrm{~Hz}$, the frequency corresponding to this $4 w_{1 / 2}$ wavelength sinusoid (Figure A-1b). We define this $4 w_{1 / 2}$ wavelength as the "Fourier" wavelength:

$$
\lambda_{f}=4 w_{1 / 2}=3.1 z_{c} .
$$

We use wavelength to estimate the upper limit of the gravity signal waveband. To convert the Fourier wavelength into the "anomaly detection threshold" frequency, wavelength is divided by aircraft speed to yield period. Frequency is 1 /period. 